\title{
EVENTOS ADVERSOS EM UMA UNIDADE DE INTERNAÇÃO CIRÚRGICA: ESTUDO DESCRITIVO
}

\author{
Adverse events in a hospital surgical unit: a descriptive study \\ Eventos adversos en una unidad de internación quirúrgica: estudio descriptivo
}

\author{
Bruna Telemberg Sell', Lúcia Nazareth Amante², Tatiana Martins', \\ Camilla Telemberg Sell 4 , Fabiana Minati de Pinho ${ }^{5}$, Renata da Silva ${ }^{6}$
}

RESUMO: Objetivo: Verificar a ocorrência de infecção do sítio cirúrgico, perda ou infecção do acesso venoso e quedas em pacientes internados em Unidade de Internação Cirúrgica de um Hospital de Ensino. Método: Estudo descritivo de abordagem quantitativa. O período de coleta de dados foi entre 23 de março e 14 de abril de 2015, em dias alternados, totalizando 94 pacientes. Resultado: A média do tempo de internação foi de 8,46 dias, com desvio padrão de 11,294. Observou-se um total de 20 (7,9\%) eventos adversos. Houve nove (3,6\%) notificações de perda do acesso venoso, sete notificações de infecção do sítio cirúrgico $(2,8 \%)$ e quatro $(1,6 \%)$ notificações de infecção do acesso venoso. Conclusão: Observou-se que o índice de ocorrência dos eventos adversos foi superior a um estudo semelhante, evidenciando a necessidade de ações que estimulem a notificação de eventos adversos e a promoção da segurança do paciente. Palavras-chave: Enfermagem perioperatória. Segurança do paciente. Doença iatrogênica.

ABSTRACT: Objective: Verify the occurrence of infection at surgical sites, loss, or infection of venous access and falls in patients hospitalized in the Surgical Inpatient Unit of a Teaching Hospital. Method: Descriptive study with a quantitative approach. The period of data collection was between March 23 and April 14, 2015 on alternating days, totaling 94 patients. Results: The average hospitalization time was 8.46 days, with a standard deviation of 11.294 . A total of $20(7.9 \%)$ adverse events were observed. There were nine (3.6\%) records of venous access loss, seven records of surgical site infections (2.8\%), and four $(1.6 \%)$ records of venous access infection. Conclusion: It was observed that the occurrence index of the adverse events was greater than in a similar study, showing the need for actions that stimulate the recording of adverse events and the promotion of patient's safety.

Keywords: Perioperative nursing. Patient Safety. Iatrogenic disease.

RESUMEN: Objetivo: Verificar la ocurrencia de infección del sitio quirúrgico, pérdida o infección del acceso venoso y caídas de pacientes internados en Unidad de Internación Quirúrgica de un Hospital Escuela. Método: Estudio descriptivo de abordaje cuantitativo. El período de colecta de datos fue entre el 23 de marzo y el 14 de abril de 2015, en días alternados, totalizando 94 pacientes. Resultado: El promedio del tiempo de internación fue de 8,46 días, con desvío estándar de 11,294. Se observó un total de 20 (7,9\%) eventos adversos. Hubo nueve (3,6\%) notificaciones de pérdida del acceso venoso, siete notificaciones de infección del sitio quirúrgico $(2,8 \%)$ y cuatro $(1,6 \%)$ notificaciones de infección del acceso venoso. Conclusión: Se observó que el índice de ocurrencia de los eventos adversos fue superior a un estudio semejante, evidenciando la necesidad de acciones que estimulen la notificación de eventos adversos y la promoción de la seguridad del paciente.

Palabras clave: Enfermería perioperatoria. Seguridad del Paciente. Enfermedad iatrogênica

\footnotetext{
'Enfermeira. Mestre em Enfermagem. Universidade Federal de Santa Catarina (UFSC). E-mail: sellbruna@hotmail.com

Rua José Lino Kretzer, 915, apartamento 406 A - Praia Comprida - CEP: 88103-902 - São José (SC), Brasil.

${ }^{2}$ Enfermeira. Doutora. Departamento de Enfermagem da UFSC. E-mail: lucia.amante@ufsc.br

${ }^{3}$ Enfermeira. Mestre em Enfermagem. UFSC. E-mail: tatiana_martins15@hotmail.com

«Enfermeira. Mestre em Enfermagem. UFSC. E-mail: camillasell@hotmail.com

${ }^{5}$ Enfermeira. Mestre. Hospital Professor Polydoro Ernani de São Thiago. E-mail: fabiminati@yahoo.com.br

${ }^{6}$ Enfermeira. Mestre em Gestão do Cuidado em Enfermagem. Universidade Federal de Santa Catarina. E-mail: renataenf76@gmail.com

Recebido: 06 jun. 2016. Aprovado: 14 jun. 2016

DOI: $10.5327 / Z 1414-4425201600030005$
} 


\section{INTRODUÇÃO}

O sistema de saúde desenvolve ações curativas e preventivas a fim de promover a segurança do paciente; entretanto, mesmo nas melhores instituições, os pacientes apresentam riscos de serem vítimas de Eventos Adversos (EA) ${ }^{1}$.

Para fomentar o comprometimento com a promoção da segurança do paciente em todos os estados membros da Organização Mundial da Saúde (OMS), a Aliança Mundial para a Segurança do Paciente, a cada dois anos, formula os Desafios Globais para a segurança do paciente. Em 2009, a assistência cirúrgica foi elencada como área problemática para o segundo desafio global para a segurança do paciente, e foi elaborado um Manual para a Cirurgia Segura, intitulado como Cirurgias Seguras Salvam Vidas, a fim de minimizar a perda desnecessária de vidas e complicações sérias².

Apesar de a OMS lançar como área problemática a assistência cirúrgica como um todo, o segundo desafio global contempla basicamente o período operatório, com pouco ou nenhum destaque para os períodos pré- e pós-operatórios. Entretanto, outras ações são recomendadas e estimuladas pela OMS, podendo contribuir para a segurança do paciente cirúrgico em todas as suas fases, tais como: evitar a troca de pacientes ao prestar qualquer cuidado, garantir a comunicação adequada entre as equipes e evitar a má conexão de tubos e cateteres ${ }^{3}$.

Mesmo com a criação de comissões, alianças e políticas públicas visando a promoção da segurança do paciente em vários aspectos, existe a ocorrência de incidentes e EA na realidade dos serviços de saúde. Estas ocorrências podem ser impactantes para o Sistema Único de Saúde (SUS) por resultar, em algumas situações, no aumento da mortalidade, da morbidade, do tempo de tratamento dos pacientes, dos custos assistenciais, entre outras ${ }^{4}$.

Assim, em virtude deste cenário, a equipe de saúde e de enfermagem tem papel fundamental na promoção da segurança do paciente e qualidade dos serviços, o que influencia diretamente na prevenção da ocorrência dos incidentes, erros e EA 5 . A ocorrência dos EA pode ter múltiplas causas, entre elas, aquelas relacionadas ao dimensionamento dos profissionais; à formação e qualificação profissional; aos materiais e equipamentos disponíveis; às condições estruturais; ao acesso a novas tecnologias e informações; ao processo de trabalho, entre outros ${ }^{6}$.

Em relação aos EA cirúrgicos, foi realizado um estudo, por meio da revisão retrospectiva de prontuários de pacientes admitidos no ano de 2003, em três hospitais gerais, públicos e de ensino, localizados no estado do Rio de Janeiro. A amostra final foi composta com 1.103 pacientes, dos quais 18 apresentaram algum EA cirúrgico, configurando a incidência de $3,5 \%$. Entre os pacientes com EA cirúrgico, três $(7,9 \%)$ tiveram mais de um EA, resultando em uma média de 1,1 eventos por paciente, sendo que a proporção de EA cirúrgicos evitáveis foi estimada em $68,3 \%$. Os EA cirúrgicos mais recorrentes foram os relacionados com a ferida cirúrgica, que ocorreu em 46,3\% dos EA, sendo 19,5\% por infecção de ferida cirúrgica e 26,8\% por outro problema com a ferida cirúrgica. Infecção não relacionada à ferida cirúrgica ocorreu em 14,6\%, e hemorragias em $12,2 \%$ dos $\mathrm{EA}^{7}$. Alguns estudos realizados em unidades de internação cirúrgica destacaram a ocorrência de diversos EA específicos, como por exemplo quedas, retirada de sondas, drenos e cateteres e infecções do sítio cirúrgico (ISC) ${ }^{7-9}$.

Em virtude deste contexto, e ciente de que as ações da equipe de enfermagem podem prevenir a ocorrência de erros e eventos adversos (EV) promovendo a segurança do paciente, tem-se a seguinte pergunta de pesquisa: qual a ocorrência de infecção do sítio cirúrgico, perda ou infecção do acesso venoso e quedas em pacientes internados em uma Unidade de Internação Cirúrgica de um Hospital de ensino (HE)?

Sendo assim, o objetivo do estudo foi verificar a ocorrência de infecção do sítio cirúrgico, perda ou infecção do acesso venoso e quedas em pacientes internados em uma Unidade de Internação Cirúrgica de um HE.

\section{MÉTODO}

Pesquisa descritiva, quantitativa, realizada em unidade de internação cirúrgica de um hospital universitário do sul do Brasil. A coleta de dados foi realizada entre 23 de março e 14 de abril de 2015, em dias alternados. A unidade de escolha atende cirurgias de cabeça e pescoço, gastrointestinal e vias biliares, torácica, otorrinolaringologia, bucomaxilofacial, transplante hepático e neurocirurgia. No ano de 2013, 1.489 pacientes foram internados nessa unidade, com uma média mensal de aproximadamente 730 internações. Foram realizadas 2.259 cirurgias, sendo 738 cirurgias limpas, 1.248 cirurgias potencialmente contaminadas, 233 cirurgias contaminadas e 40 cirurgias infectadas ${ }^{10}$.

Para o cálculo do tamanho da amostra, considerouse o número de pacientes internados nesta unidade cirúrgica em 2013, dado obtido do Boletim Epidemiológico da Comissão de Controle de Infecção Hospitalar, que foi de 1.489 pacientes $^{10}$. Foi utilizado o programa computacional 
de ensino-aprendizagem de estatística, o SestatNet, resultando em uma amostra de 94 pacientes, com nível de confiança de $95 \%$. Fizeram parte deste estudo todos os pacientes, de ambos os sexos, maiores de 15 anos, internados na Unidade de Internação Cirúrgica I (UIC I) até atingir o número mínimo de 94 pacientes.

Foram utilizados quatro instrumentos para a coleta de dados:

1. Roteiro para caracterização dos pacientes e identificação dos EA;

2. Roteiro para avaliação da ocorrência de queda;

3. Roteiro para avaliação da ocorrência de ISC;

4. Roteiro para avaliação da ocorrência de perda ou infecção do acesso venoso.

A validação dos instrumentos deu-se por meio de um pré-teste realizado com 28 pacientes, previamente à coleta de dados, cujos resultados não foram utilizados para a análise de dados. Estes instrumentos foram construídos a partir dos conceitos e estudos encontrados na revisão de literatura.

Nos dias de coleta de dados, todos os pacientes internados foram avaliados e entrevistados a fim de se identificar a ocorrência dos EA. Nesse sentido, foram feitas 252 avaliações nos 94 pacientes selecionados.

Os dados foram registrados no software estatístico Statistical Package for the Social Sciences (SpSS). Para a análise das variáveis categóricas, utilizou-se a distribuição de frequência e o teste do $\chi^{2}$, e para as variáveis quantitativas utilizou-se média, desvio-padrão (DP) e a ANOVA com um fator fixo. Para as análises inferenciais, o nível de significância adotado foi de $5 \%$.

O projeto foi submetido ao Comitê de Ética e Pesquisa com Seres Humanos da Instituição de Ensino de origem, e recebeu parecer favorável - Certificado de Apresentação para Apreciação Ética 39652314.6.0000.0115.

\section{RESULTADOS}

Os dados encontrados foram analisados e constituíram três categorias para análise, quais sejam: dados demográficos dos pacientes; ocorrência de infecção do sítio cirúrgico; ocorrência da perda do acesso venoso e ocorrência de infecção do acesso venoso.

\section{Dados demográficos dos pacientes}

Observou-se uma variação de idade entre 15 e 83 anos, sendo a média de idade dos pacientes de 50,04 anos com DP de 16,928.
Em relação ao sexo, 57 pacientes $(60,6 \%)$ eram mulheres e $37(39,4 \%)$ eram homens. Avaliando o estado civil, constatou-se que 51 deles $(54,3 \%)$ eram casados, 21 (22,3\%) eram solteiros, $12(12,8 \%)$ eram viúvos, $6(6,4 \%)$ divorciados e 4 (4,3\%) viviam em união estável. Observando a escolaridade, constatou-se que $27(28,7 \%)$ pacientes possuíam ensino fundamental incompleto; $26(27,7 \%)$ com ensino médio completo; 14 (14,9\%) possuíam ensino fundamental completo; 10 (10,6\%) possuíam superior incompleto; 8 (8,5\%) com ensino médio incompleto; $7(7,4 \%)$ possuíam superior completo; e, $2(2,1 \%)$ pacientes não eram alfabetizados.

O tempo de internação dos pacientes variou de 0 a 59 dias, sendo a média do tempo de internação de 8,46 dias, com DP de 11,294.

Os motivos que levaram os pacientes à internação foram diversos, sendo mais prevalentes as internações para Colecistectomia Vídeo Laparoscópica (CVL) e para Colangiopancreatografia Endoscópica Retrógrada (CPRE). Observou-se também internações por Apendicite, Hernioplastia Inguinal, Gastroplastia e Tireoidectomia. Com um menor valor de prevalência, foram observados Câncer Gástrico e Paroidectomia. É possível visualizar estes dados na Figura 1.

Em relação à presença de infecção anterior à data da internação, observou-se que $76(80,9 \%)$ dos pacientes não apresentaram nenhum tipo de infecção prévia e que 18 (19,1\%) pacientes internaram com algum tipo de infecção prévia.

Em relação à ocorrência dos EA, ISC, à perda do acesso venoso, à infecção do acesso venoso e às quedas

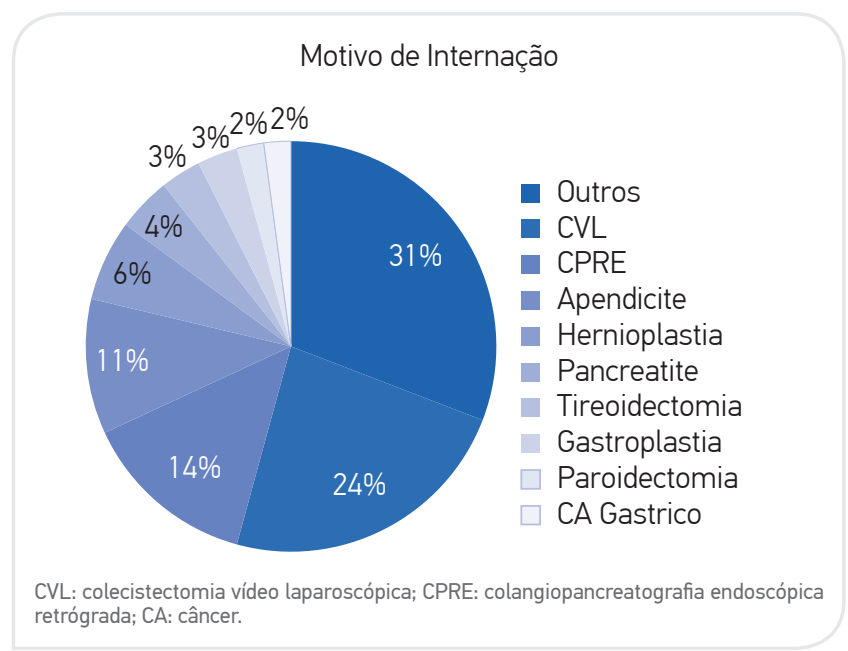

Figura 1. Porcentagem dos Motivos de Internação. Florianópolis, Santa Catarina, Brasil, 2015. 
nas 252 avaliações, observou-se um total de 20 (7,9\%) EA. Houve nove $(3,6 \%)$ notificações de perda do acesso venoso, sete $(2,8 \%)$ notificações de ISC, quatro (1,6\%) notificações de infecção do acesso venoso, e não houve ocorrência de queda (Figura 2).

\section{Ocorrência de infecção do sítio cirúrgico}

Os pacientes que desenvolveram ISC tinham uma média de idade de 46,7 anos, sendo quatro deles do sexo feminino e três do sexo masculino, em sua maioria com baixo grau de escolaridade. Os motivos de internação foram: Abcesso Hepático, Abdômen Agudo, Colecistite, Apendicite, Laparotomia Exploradora, Carcinoma de Células Hepáticas e Coledocolitíase.

A média do tempo de internação pré-operatória dos sete pacientes que desenvolveram ISC foi de 4,86 dias, com DP de 7,493. O tempo mínimo de internação pré-operatória foi de zero dias, e o tempo máximo foi de 21 dias.

Em relação ao grau de contaminação da cirurgia dos pacientes que desenvolveram ISC, dois realizaram cirurgia considerada limpa, três praticaram cirurgia considerada potencialmente contaminada e dois cirurgia considerada contaminada. Em relação ao tipo de cirurgia dos pacientes que desenvolveram ISC, três cirurgias foram eletivas e quatro foram de emergência. Em relação ao porte da cirurgia dos pacientes que desenvolveram ISC, duas cirurgias foram de pequeno porte, três cirurgias foram de médio porte e duas de grande porte. Em relação ao risco cirúrgico todos os pacientes que desenvolveram ISC apresentaram ASA II - pontuação para avaliação subjetiva de saúde geral do paciente pela American society of Anesthesiologists (ASA).

Em relação à classificação da ferida dos pacientes que desenvolveram ISC, seis feridas foram classificadas como infecções incisionais profundas e uma como infecção de órgão/ espaço.

Após a primeira notificação de ISC, as feridas foram acompanhadas e avaliadas em sua evolução, resultando em 24 avaliações de feridas infectadas. A Figura 3 a seguir demonstra a presença de sinais flogísticos durante as avaliações das feridas operatórias infectadas:

A dor foi mencionada em 9 (37,5\%) avaliações e não foi mencionada em 15 (62,5\%) delas; o rubor foi identificado em $3(12,5 \%)$ avaliações e não foi identificado em 21 (87,5\%) delas; o edema foi verificado em 6 (15\%) avaliações e ausente em 18 (75\%); e a febre foi ausente em todas elas.

Em relação à presença de deiscência de sutura durante as avaliações das feridas infectadas, observou-se 4 (16,6\%) feridas com ausência de deiscência e 20 (83,3\%) com deiscência. Em relação à presença de exsudato na ferida infectada, foi constatada a presença de exsudato em todas as feridas.

Quanto à presença de dreno nos pacientes com ISC, foi observada a ausência de dreno em 11 (45,8\%) avaliações, $10(41,6 \%)$ com dreno de penrose, e 3 (12,5\%) com dreno tubular. Sobre o tipo de exsudato do dreno, observou-se que em $11(45,8 \%)$ avaliações o exsudato era ausente, em 5 $(20,8 \%)$ a drenagem era purulenta, em $3(12,5 \%)$ a drenagem era serosanguinolenta, em $2(8,3 \%)$ a drenagem era biliosa, em $2(8,3 \%)$ a drenagem era serosa e em $1(4,1 \%)$ a drenagem era seropurulenta.

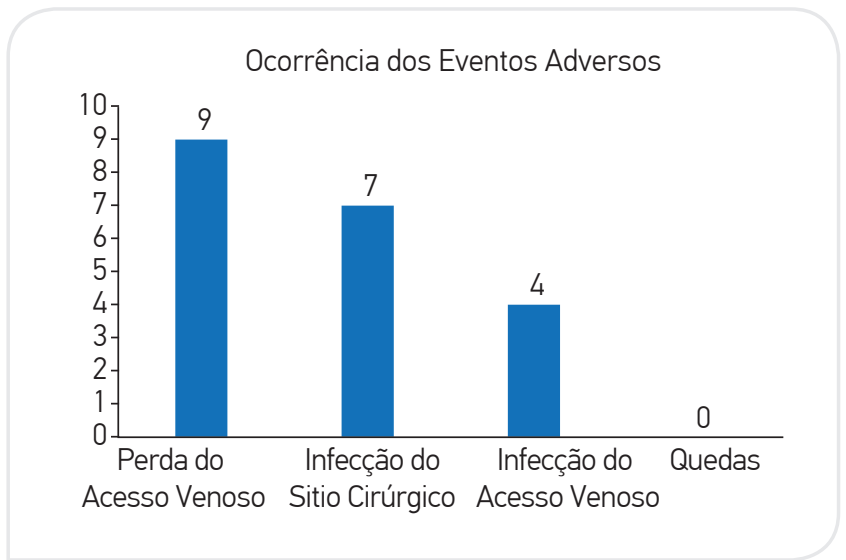

Figura 2. Frequência da ocorrência dos eventos adversos nas 252 avaliações. Florianópolis, Santa Catarina, Brasil, 2015.

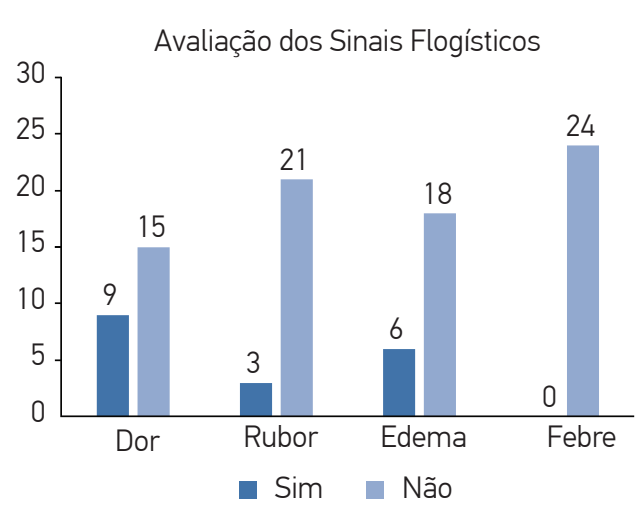

Figure 3. Presença ou ausência de Sinais flogísticos nas Infecções do Sítio Cirúrgico. Florianópolis, Santa Catarina, Brasil, 2015. 


\section{Ocorrência da perda do acesso venoso}

Constatou-se neste estudo que nas nove ocorrências de perda do acesso venoso, o tipo de acesso era periférico, portanto não houve a ocorrência de perda de acesso venoso central em nenhuma das observações.

Em relação ao tempo de punção venosa, em dias, nas ocorrências de perda do acesso venoso, não se observou diferença estatisticamente significativa. Em três ocorrências, o tempo de punção venosa foi de um dia; em duas ocorrências, o tempo foi de dois dias; em duas ocorrências, o tempo foi de três dias; e em duas ocorrências o tempo de punção não foi registrado pela equipe de enfermagem.

Em relação à troca de curativo do acesso venoso, notou-se que $100 \%$ das trocas de curativo foram realizadas a cada 72 horas, por profissionais da enfermagem de nível médio, utilizando como antisséptico a clorexidina alcoólica $0,5 \%$. E, em relação à fixação do cateter, cinco foram fixados com micropore e quatro com película transparente.

No que tange ao local da punção venosa, na ocorrência de perda do acesso venoso, observou-se que quatro punções foram no braço, três no antebraço, uma na fossa anticubital e uma no dorso da mão. Em relação à coloração da pele na ocorrência de perda do acesso venoso, observaram-se cinco pacientes com cor de pele branca, dois pacientes com cor de pele parda e dois pacientes com cor de pele negra.

Ao observar o ambiente da ocorrência da perda do acesso venoso, constatou-se que em seis ocorrências o suporte de soro era fixado na parede e em três, o suporte de soro era com rodízio. Em relação ao tipo de infusão venosa, percebeu-se que em sete ocorrências a infusão era contínua e em duas a infusão era lenta.

Em relação à solução infundida no acesso venoso, observou-se a presença de solução fisiológica em nove ocorrências e antibiótico em quatro ocorrências, sendo ausente a infusão de soluções glicosadas a 5, 10 e 50\%; ringer; ringer lactato; drogas vasoativas; antifúngicos e nutrição parenteral. Ao observar os dispositivos utilizados no acesso venoso, percebeu-se que oito deles utilizavam extensores intermediários e um utilizava apenas equipo com injetor lateral. Em relação ao tamanho do cateter sob a agulha utilizada na punção venosa, oito eram de calibre 24 e uma de calibre 22.

Em relação à presença dos sinais flogísticos, observou-se dor local em quatro ocorrências e rubor em quatro ocorrências. A flebite, a febre, e o exsudato purulento estavam ausentes.

\section{Ocorrência de infecção do acesso venoso}

Ao analisar as ocorrências de infecção do acesso venoso, observou-se que das quatro ocorrências de infecção do acesso venoso, o tipo de acesso era periférico, não havendo a ocorrência de perda de acesso venoso central em nenhuma das observações.

Em relação ao tempo de punção venosa em dias, nas ocorrências de infecção do acesso venoso, observou-se que em duas ocorrências o tempo de punção venoso era de um dia, em uma ocorrência era de dois dias e em uma ocorrência o tempo de punção venoso era de três dias, evidenciando ausência de diferença estatisticamente significativa.

Ao observar a frequência da troca de curativo do acesso venoso, constatou-se que em $100 \%$ das ocorrências a troca do curativo era realizada a cada 72 horas, por profissionais da enfermagem de nível médio, utilizando como antisséptico a clorexidina alcoólica $0,5 \%$. Em relação à fixação do cateter, uma delas foi com micropore e três com película transparente.

Sobre o local da punção venosa na ocorrência de infecção do acesso, observaram-se três punções no braço e uma no antebraço. Em relação à coloração da pele na ocorrência de infecção do acesso venoso, observaram-se dois pacientes com cor branca, um paciente de cor parda e um paciente negro.

Observando o ambiente em que ocorreu a infecção do acesso venoso, constatou-se que em três ocorrências o suporte de soro era fixado na parede, e em uma o suporte de soro era com rodízio. Em relação ao tipo de infusão venosa, percebeu-se que em três ocorrências a infusão era contínua e em uma a infusão era lenta. Em relação à solução infundida no acesso venoso, observou-se a presença de solução fisiológica em quatro ocorrências e antibiótico em uma ocorrência. Sendo, portanto, ausente a infusão de soluções glicosadas a 5, 10 e 50\%, ringer, ringer lactato, drogas vasoativas, antifúngicos e nutrição parenteral. Ao observar os dispositivos utilizados no acesso venoso, percebeu-se que três utilizam extensores intermediários e uma utilizava apenas equipo com injetor lateral. Em relação aos cateteres utilizados na punção venosa, todos eram do calibre 24 .

Em relação à presença dos sinais flogísticos, observou-se dor local em quatro ocorrências e rubor em quatro ocorrências. Estavam ausentes a flebite e febre e o exsudato purulento.

Observou-se que houve uma simultaneidade entre a perda do acesso venoso e a ocorrência de infecção do acesso venoso entre quatro pacientes. 


\section{DISCUSSÃO}

A média de tempo de internação dos pacientes foi de 8,46 dias. Segundo a Agência Nacional de Saúde Suplementar (ANS), este dado é um indicador de desempenho hospitalar, e uma média acima de 7,0 dias de internação em hospitais agudos aumenta o risco de infecção hospitalar. Este mesmo documento indica que alguns hospitais gerais, sem atividade de ensino, relataram média de internação dos pacientes entre 3,5 e 6,4 dias $^{11}$. Portanto, neste HE, a média do tempo de internação foi superior ao indicado pela ANS e maior que outros hospitais gerais sem atividade de ensino.

Sendo assim, podemos considerar que este período prolongado de internação pode estar relacionado à característica do hospital em estudo que desenvolve atividade de ensino e à especificidade de tratamento realizado em clínica cirúrgica, no qual os pacientes encontram-se em momentos particulares que constituem a experiência cirúrgica, como os períodos pré, intra e pós-operatórios ${ }^{12}$. Portanto, a maioria dos pacientes foi internada por situações pontuais e cirúrgicas e, alguns dias após a cirurgia, os pacientes receberam a alta hospitalar.

Em relação ao índice de ocorrência dos EA cirúrgicos, observou-se que este foi superior ao de um estudo semelhante, realizado em três hospitais gerais públicos e de ensino, em que a incidência dos 1.103 pacientes com EA cirúrgicos foi 3,5\% ${ }^{7}$.

Entre os EA cirúrgicos, destaca-se a ISC, que está contida no cenário das infecções hospitalares, conceituada como qualquer infecção adquirida após a internação e que se manifesta durante a internação ou após a alta hospitalar quando relacionado com a internação ou procedimentos hospitalares ${ }^{13}$. Mesmo sendo o EA cirúrgico mais característico, a incidência de ISC neste estudo foi pequena quando comparada a outro estudo que obteve um índice de $3,68 \%$ de prevalência em um total de 2.203 pacientes. Contudo, observou-se semelhança em relação à classificação do risco cirúrgico, sendo o mais prevalente o ASA II, assim como em relação às características dos pacientes que apresentavam baixa escolaridade, eram do sexo feminino, tinham idade média de 48 anos e foram submetidos a CVL e Laparotomia Exploradora ${ }^{14}$.

Os motivos para interrupção e complicações relacionados à terapia intravenosa podem ser decorrentes de diversos fatores, inclusive podendo ocorrer de maneira simultânea, como foi observado nesta pesquisa, assim como em um estudo realizado em 2011 que indica que a incidência de flebite em adultos hospitalizados foi em média cinco vezes maior que a aceita pela Infusion Nurses Society ${ }^{15}$.

Neste estudo, o EA prevalente foi a perda do cateter venoso periférico, sendo constado que das nove perdas, quatro foram resultantes da infecção do acesso venoso. Um estudo realizado em 2011, que envolveu 76 pacientes internados em uma enfermaria, observou prevalência na ocorrência de flebite em $25,8 \%$ dos pacientes com cateter intravenoso periférico, sendo que destes, $40 \%$ apresentaram manifestações clínicas como dor, edema e eritema. Neste estudo, foi possível observar as manifestações clínicas dor e eritema em quatro observações de infecção do acesso venoso. Houve semelhança também em relação ao tempo de permanência do cateter intravenoso, que em ambas as pesquisas, foi inferior ou menor a 72 horas $^{15}$.

Não houve a ocorrência de queda durante os dias de coleta de dados; entretanto, há evidências em outros estudos da frequência e relevância deste EA no âmbito hospitalar e cirúrgico. Um estudo realizado em 2010 demonstra que $10,7 \%$ dos boletins de notificação de EA estão relacionados a quedas, resultando em uma média mensal de 2,6 quedas ${ }^{16}$, assim como no estudo realizado em 2014, que evidencia a ocorrência de 93,5\% das quedas em Unidade de Internação Cirúrgica e 6,5\% no Serviço de Emergência ${ }^{17}$.

Neste contexto, a equipe de enfermagem tem papel fundamental na prevenção de EA em Unidade de Internação Cirúrgica, com destaque para a ocorrência de ISC, assim como na redução das complicações relacionadas ao acesso venoso periférico, tendo em vista que este procedimento é de responsabilidade da enfermagem e afeta a integridade cutânea do paciente, aumentando o risco de infecção. A prevenção da infecção relacionada ao cateter venoso periférico é, principalmente, de responsabilidade da equipe de enfermagem, refletindo na qualidade da assistência prestada e segurança do paciente, além de reduzir o tempo de internação e os custos ${ }^{18}$.

Uma maneira de diminuir a distância entre o cuidado de enfermagem ideal e o cuidado de enfermagem real é trabalhar nas equipes de saúde a ocorrência do erro e a culpa profissional, aproveitando esta oportunidade para discutir e envolver os profissionais no pensar crítico sobre as ações de cuidado e atitudes em relação ao próprio erro ou ao erro do colega, utilizando as ocorrências para um momento de aprendizado, e, dessa forma, evitando novos erros relacionados à mesma causa ${ }^{19}$.

Neste sentido é essencial modificar, nos ambientes hospitalares, a forma de aceitar os erros como falhas individuais 
e encorajar a notificação dos erros. Igualmente, a assistência deve ser centrada no paciente, baseada em modelos que facilitam o trabalho em equipe, colaborativo e interdisciplinar ${ }^{3}$.

Concomitantemente a isso, é necessário que as instituições e os gestores estejam comprometidos com a promoção da segurança do paciente, estabelecendo-a como um eixo norteador de sua organização. Portanto, é fundamental oferecer subsídios aos prestadores de cuidado direto, buscando conhecer as dificuldades e desafios da assistência para que seja possível uma comunicação efetiva entre todos os níveis hierárquicos que possibilite a confiança entre os profissionais envolvidos ${ }^{19}$.

A promoção da segurança do paciente deve estar alinhada a uma cultura institucional e profissional de segurança, sendo necessário que ocorram nos ambientes hospitalares algumas mudanças, a saber: mudar a busca de erros como falhas individuais para compreendê-los como causa de falhas do sistema; mudar de um ambiente punitivo para uma cultura na qual a notificação do erro é estimulada; evitar o sigilo evidenciando a transparência; centralizar o cuidado no paciente e não no médico; não utilizar modelos de cuidado baseados na excelência do desempenho individual e independente, mas sim em modelos de cuidado realizados por equipe profissional interdependente, colaborativos e interdisciplinares; prestação de contas universal e recíproca, e não do topo para a base $e^{3}$.

neste sentido, a criação de uma cultura de segurança do paciente dentro das instituições de saúde permite que as equipes se sintam mais seguras ao informar a ocorrência de incidentes e EA, pois viabiliza estas mudanças ${ }^{20}$.

Ainda assim, observa-se como limitação deste estudo o período de coleta de dados, que para obtenção de dados mais consistentes e impactantes precisaria ser maior, resultando em maior número de avaliações.

\section{CONCLUSÃO}

Ficou evidente que a presença de EA no ambiente cirúrgico deste HE é relevante quando comparado a estudos semelhantes, em que o índice da ocorrência dos EA foi menor do que neste estudo. Entre estes eventos observados, o mais recorrente foi a perda do acesso venoso, seguido de ISC e infecção do acesso venoso. É interessante destacar que não houve ocorrência de quedas no período de coleta de dados, porém não se pode excluir a possibilidade desta ocorrência em unidade de internação cirúrgica, tendo em vista o tempo reduzido de coleta de dados neste estudo.

A presença de EA em uma Unidade de Internação Cirúrgica evidencia falhas na qualidade da assistência prestada, portanto, após a ocorrência, os EA devem ser analisados para elucidar as possíveis causas e permitir reflexões e momentos para educação permanente à equipe de saúde e de enfermagem, objetivando medidas que promovam a prevenção e a diminuição de erros.

A promoção de uma cultura de segurança deve ser estabelecida entre os profissionais para que a notificação dos incidentes, erros e EA, não denote caráter punitivo ao profissional, mas sim entendimento de que o erro é multifatorial e não de responsabilidade de um único profissional.

É necessário que sejam investigadas as principais causas da ocorrência dos EA para que seja possível a realização de ações que visem sua prevenção, como educação permanente, quantitativo profissional adequada, dispositivos e materiais em condições ótimas, rotinas bem estabelecidas, protocolos clínicos elaborados, procedimento operacional padrão condizente com a realizada institucional e de fácil acesso, instrumento de notificação de EA disponíveis à equipe.

\section{REFERÊNCIAS}

1. Pedreira MLG. Nursing interventions and outcomes to ensure patient's safety. Acta Paul Enferm. 2009;22(4):v-vi. [acesso em 10 dez 2015]. Disponível em: http://www.scielo.br/ scielo.php?pid=S0103-21002009000400001\&script=sci_ arttext\&tlng=en

2. Organização Mundial de Saúde. Segundo desafio global para a segurança do paciente: Cirurgias seguras salvam vidas. Rio de
Janeiro (RJ): Organização Pan-Americana da Saúde/Ministério da Saúde/Agência Nacional de Vigilância Sanitária; 2009. [citado 2015 jun. 20]. Disponível em: http://bvsms.saude.gov.br/bvs/publicacoes/ seguranca_paciente_cirurgia_salva_manual.pdf

3. Brasil. Portaria n 529, de 10 de abril de 2013: Institui o Programa Nacional de Segurança do Paciente (PNSP). Diário Oficial da República Federativa do Brasil, 02 abr. 2013. Seção 1. 
4. Brasil. Agência Nacional de Vigilância Sanitária. Investigação de eventos adversos em serviços de saúde. Série Segurança do paciente e qualidade em serviços de saúde. Brasília: ANVISA; 2013. [citado 2015 jun. 20]. Disponível em: http://www20.anvisa. gov.br/segurancadopaciente/images/documentos/livros/Livro5InvestigacaoEventos.pdf

5. Bohomol E, Tartali JA. Adverse effects in surgical patients: knowledge of the nursing professionals. Acta Paul Enferm. 2013;26(4):37681. [acesso em 19 set 2015]. Disponível em: http://www.scielo. br/scielo.php?pid=S0103-21002013000400012\&script=sci_arttext \&tlng=en

6. Arboit EL, Silva LAA. Eventos adversos na enfermagem relacionados ao dimensionamento de pessoal. Rev Enferm. 2012;8(8):128-39. [acesso em 28 abr 2015]. Disponível em: http://www.revistas.fw.uri. br/index.php/revistadeenfermagem/article/view/481/877

7. Moura MLO, Mendes W. Assessment of surgical adverse events in Rio de Janeiro hospitals. Rev Bras Epidemiol. 2012;15(3):523-35. [acesso em 20 jun 2015]. Disponível em: http://www.scielo.br/ scielo.php?pid=S1415-790X2012000300007\&script=sci_arttext \&tlng=en

8. Carneiro FS. Eventos adversos na clínica cirúrgica de um hospital universitário: instrumento de avaliação da qualidade. Rev Enferm. 2011;19(2):204-11. [acesso em 20 jun 2015]. Disponivel em: http:// www.facenf.uerj.br/v19n2/v19n2a06.pdf

9. Oliveira AC, Braz NJ, Ribeiro MM. Incidência da infecção do sitio cirúrgico em um hospital universitário. Cienc Cuid Saúde. 2007;6(4):486-93. [acesso em 20 out 2015]. Disponível em: http://eduem.uem.br/ojs/ index.php/CiencCuidSaude/article/view/3685/2687

10. Masukawa II, Vieira GB, Klein TR. Boletim Epidemiológico - Serviço de Controle de Infecção Hospitalar (SClH/HU). $5^{\circ}$ Título. Florianópolis (SC); 2014.

11. Agência nacional de saúde suplementar. Média de permanência geral. Ministério da Saúde; 2013 [citado 2016 sep. 01]. Disponível em: http://www.ans.gov.br/images/stories/prestadores/E-EFI -05.pdf

12. Cheregatti AL. Enfermagem em clínica cirúrgica no pré e no pósoperatório. São Paulo (SP): Martinari; 2012.
13. Brasil. Portaria n० 930/MS, de 27 de agosto de 1992. Resolve expedir, na forma de seus anexos, normas para o controle das infecções hospitalares. Diário Oficial da República Federativa do Brasil, 04 set. 1992. Seção 1.

14. Aguiar APL, Prado PR, Opitz SP, Vasconcelos SP, Faro ARMC. Fatores associados à infecção do sitio cirúrgico em um hospital na Amazônia ocidental brasileira. Rev SOBECC. 2012;17(3):60-70. [acesso em 20 jun 2015]. Disponível em: http://itpack31.itarget.com.br/uploads/snf/arquivos/ Ano17_n3_\%20jul_set2012_(Fatores_associados_infeccao_de_sitio_ cirurgico_em_um_hospital_na_amazonia_ocidental_brasileira).pdf

15. Magerote NP, Lima MHM, Silva JB, Correia MDL, Secoli SR. Associação entre flebite e retirada de cateteres intravenosos periféricos. Texto Contexto - Enferm. 2011;20(3):486-92. [acesso em 21 ago 2015]. Disponível em: http://www.scielo.br/scielo. php?script=sci_arttext\&pid=S0104-07072011000300009

16. Paiva MCMS, Paiva SAR, Barti HW, Campana AO. Caracterização das quedas de pacientes segundo notificação em boletins de eventos adversos. Rev Esc Enferm. 2010;44(1):134-8. [acesso em 21 ago 2015]. Disponível em: http://www.scielo.br/pdf/reeusp/v44n1/a19v44n1.pdf

17. Prates CG, Luzia MF, Ortolan MR, Neves CM, Bueno ALM, Guimarães F. Quedas em adultos hospitalizados: incidência e características desses eventos. Cienc Cuid Saúde. 2014;13(1):74-81. [acesso em 10 dez 2015]. Disponível em: http://www.periodicos.uem.br/ojs/index. php/CiencCuidSaude/article/view/20728/pdf_115

18. Mendonça KM, Neves HCC, Barbosa DFS, Souza ACS, Tipple AFV, Prado MA. Atuação da enfermagem na prevenção e controle de infecção de corrente sanguínea relacionada ao cateter. Rev Enferm. 2011;19(2):330-33. [acesso em 20 jun 2015]. Disponível em: http:// www.facenf.uerj.br/v19n2/v19n2a26.pdf

19. Paese F, Dal Sasso GTM. Patient safety culture in primary health care. Texto Contexto - Enferm. 2013;22(2):302-10. [acesso em 21 ago 2015]. Disponível em: http://www.scielo.br/scielo. php?pid=S0104-07072013000200005\&script=sci_arttext\&tlng=en

20. Nascimento CCP, Toffoletto MC, Gonçalves LA, Freitas WG, Padilha KG. Indicators of healthcare results: analysis of adverse events during hospital stays. Rev Latino-am Enfermagem. 2008;16(4):74651. [acesso em 21 ago 2015]. Disponível em: http://www.scielo.br/ scielo.php?script=sci_arttext\&pid=S0104-11692008000400015 